Questions vives

\section{Questions Vives}

Recherches en éducation

$N^{\circ} 28 \mid 2017$

De l'indifférenciation à la différenciation

\title{
Enjeux d'une praxis textuelle en éducation : réflexion sur l'apport des sciences de la culture en enseignement-apprentissage des langues
}

\section{Nadia Makouar et Maryvonne Holzem}

\section{OpenEdition}

Journals

Édition électronique

URL : http://journals.openedition.org/questionsvives/2843

DOI : 10.4000/questionsvives.2843

ISSN : $1775-433 \mathrm{X}$

Éditeur

Université Aix-Marseille (AMU)

Édition imprimée

Date de publication : 29 décembre 2017

ISBN : 978-2-912643-52-0

ISSN : $1635-4079$

\section{Référence électronique}

Nadia Makouar et Maryvonne Holzem, « Enjeux d'une praxis textuelle en éducation : réflexion sur l'apport des sciences de la culture en enseignement-apprentissage des langues », Questions Vives [En ligne], $N^{\circ} 28$ | 2017, mis en ligne le 15 novembre 2018, consulté le 01 mai 2019. URL : http:// journals.openedition.org/questionsvives/2843; DOI : 10.4000/questionsvives.2843

Ce document a été généré automatiquement le 1 mai 2019.

\section{cc) (†)}

Questions Vives est mis à disposition selon les termes de la licence Creative Commons Attribution -

Pas d'Utilisation Commerciale - Pas de Modification 4.0 International. 


\title{
Enjeux d'une praxis textuelle en éducation : réflexion sur l'apport des sciences de la culture en enseignement-apprentissage des langues
}

\author{
Nadia Makouar et Maryvonne Holzem
}

\section{Introduction}

1 Cet article propose une réflexion théorique et pratique sur l'apport de la sémantique des textes en éducation. Les enjeux d'une pratique des textes impliquent une rupture avec la vision utilitariste de la langue. Ils redonnent au texte sa dimension philologique, qui exige son établissement au sein d'une lignée de réécriture, et herméneutique, posant ainsi son interprétation comme partie prenante de l'expérience humaine appréhendée dans sa singularité. Notre point de vue scientifique sera celui des sciences de la culture qui appelle, avec l'apport de Cassirer puis de Rastier, à s'engager sur l'axe de la médiation symbolique. Axe praxéologique sur lequel l'apprenant s'individue en même temps que son milieu (le monde que je constitue en même temps qu'il me constitue).

2 Après avoir rappelé notre ancrage théorique, cet article propose de revenir sur la notion de " compétences » qui est au centre des préconisations du Cadre européen commun de référence pour les Langues (CECR), compromettant la démarche critique de l'individu via une catégorisation fermée des aptitudes langagières à acquérir.

3 Notre propos a pour but de développer une réflexion sur l'esprit critique, la rationalité qui s'accorde mal avec les rythmes accélérés de l'économie, la sensibilisation et la responsabilisation en éducation car «l'enjeu de la pédagogie n'est pas seulement d'énumérer des faits - fussent-ils promus au rang de connaissances - mais d'unifier les valeurs et faits: les objets culturels sont précisément, tout à la fois, des faits et des 
valeurs " (Rastier, 2013, p. 45). Les valeurs telles qu'elles sont exprimées et contestées dans les textes ressortissent aux sciences de la culture. Elles rappellent que la transmission n'est pas simple tradition mais recontextualisation et transformation incessante et qu'elle est fondée sur une éducation du don et du contre-don dans la lignée des travaux de Marcel Mauss (1924). Cette dualité compétences/connaissances tient aussi à la définition de la langue au sein du contexte didactique et pédagogique. La langue et son lieu de production doivent donc retrouver toute leur place et ce, surtout à l'heure du tout numérique et du flux informationnel incessant; l'enjeu d'une telle démarche est capital. Pour illustrer notre propos, notre article s'achèvera par une expérience pédagogique innovante menée en didactique de l'arabe ayant pour objectif de proposer à des étudiants des exercices d'analyse interprétative de textes médiatiques avec les outils d'analyse de corpus.

\section{Apport de la sémantique des textes et des sciences de la culture à une reconsidération de la notion de connaissance}

4 En linguistique saussurienne, deux aspects relatifs à la détermination et la construction du sens d'un mot sont à considérer. D'une part, le sens ne peut être étudié à partir des unités atomisées que sont les mots, le sens d'un mot étant ce que les autres ne sont pas... Ce principe différentiel conduit «à ne plus reconnaître de signes autonomes qui préexisteraient à leur combinaison » (Rastier, 2015, p. 77). Saussure écrit en effet qu'« il n'y a dans la langue ni signes, ni significations, mais des Différences de signes et des Différences de significations lesquelles $1^{\circ}$ n'existent les unes absolument que par les autres, (dans les deux sens), et sont donc inséparables et solidaires; mais $2^{\circ}$ n'arrivent jamais à se correspondre directement» (Saussure, 2002, p.70). D'autre part, la construction du sens ne saurait se limiter à l'empan de la phrase, car c'est au niveau des pratiques orales comme écrites qu'il se constitue et surtout se transforme. Cela revient à admettre que les textes en tant que suite linguistique attestée, produite dans une pratique sociale déterminée et fixée sur un support quelconque ${ }^{1}$, sont l'objet empirique de notre discipline.

5 Si nous pensons utile de rappeler cette position différentielle c'est qu'elle détourne de toute démarche ontologique et de toute théorie de la connaissance, ne traitant ni de représentation ni des objets du monde, mais de la description du sens comme «produit de différences entre signes et autres unités, tant en contexte qu'au sein des textes et des corpus» (Rastier, 2017). En conséquence, cette approche différentielle accorde le primat au concept de valeur - déterminée par la position des unités dans le système (donc par les différences). Ce qui revient à se détourner du fixisme des significations comme de l'atomisation substantivée de la chose désignée par le mot: «rien ne préexiste à la détermination de la valeur par le système. Ainsi, la valeur n'est pas un signe, mais une relation entre signifiés. Elle exclut une définition atomiste du signe, qui le pourvoirait $a$ priori d'une signification car «la signification est le résultat d'un processus d'interprétation, non une donnée » (Rastier, 2006).

Considérer le sens comme résultat de l'interprétation dans la lignée des écrits de Saussure comme de Hjelmslev appelle à prendre en compte les processus de transformation des représentations partagées que sont les valeurs telles qu'elles sont exprimées et contestées 
dans les textes et autres performances sémiotiques. Nous rejoignons ici le point de vue des sciences de la culture telles que définies par Rickert (1899) comme méthodes particularisantes en complément aux méthodes généralisantes des sciences de la nature, point de vue repris et approfondi par Cassirer (1991) dans Logique des sciences de la culture. $\mathrm{Au}$ risque de paraître trop sommaire, disons que le mot culture renvoie à la connaissance appréhendée dans l'intersubjectivité. En effet, car si la connaissance est action singulière (un individu apprenant), elle ne se fige en aucun de nous puisqu'elle n'est connaissance que reçue par un autre qui l'introduit dans sa vie. C'est à ce niveau d'intersubjectivité en acte et dans une dimension d'interprétation historique (le temps culturel non réductible à la chronologie) que Cassirer définira la spécificité des sciences de la culture. De ce point de vue, c'est donc l'usage (les échanges sur l'axe de la transmission culturelle) qui détermine la valeur et non la valeur qui détermine l'usage (à l'instar de la monnaie frappée). Les objets culturels ne relèvent pas de l'ordre des choses mais de celui des valeurs : valeurs non marchandes a contrario des spéculations qui règnent sans partage sur les marchés de l'art.

7 Pour échapper à la logique marchande, François Rastier, dans le livre qu'il consacre à l'éducation (Apprendre pour transmettre, l'éducation contre l'idéologie managériale, 2013), s'appuie sur la prégnance des notions de don et de contre-don, sur lesquelles repose la cohésion des civilisations, en référence aux écrits du sociologue Marcel Mauss (1923-1924). Pour Mauss, le don est un acte animé par une force qui n'est ni valeur matérielle, ni valeur économique. Dans l'histoire des civilisations, il a souvent pris la figure du sacrifice avec des dieux ou des esprits. Il constitue un échange fondamental intergénérationel, qui place l'éducation au cœur du processus de transmission. Le plus fondamental de tous les dons est celui de la vie et de l'éducation que reçoivent les enfants. L'enfant encourt alors une dette symbolique vis-à-vis de ses parents et de la société, dette dont il s'acquittera à son tour en transmettant lui-même ses connaissances aux enfants comme à ses contemporains. Ajoutons, toujours en référence à l'ouvrage de Rastier, que la transmission n'est pas simple tradition mais programme humaniste primordial au sein duquel l'échange symbolique exemplifie la société en acte et qui reconnaît le caractère culturellement situé de toute activité de connaissance.

8 Nous avons souhaité expliciter, sans doute trop brièvement ici, notre conception différentielle partagée, pour mieux aborder la problématique du rapport connaissances/ compétences essentielle à notre propos. La connaissance n'est donc ni accumulation stockée d'informations (domaine de l'ingénierie des connaissances), sur un support quelconque, ni objet de restitution, à l'instar d'un produit manufacturé et précalibré en fonction des attendus d'un marché.

\section{Connaissances et compétences : en sciences de l'éducation et en didactique des langues}

\subsection{Pour une dualité connaissances/compétences}

Pour décrire les aspects relevant des notions de «compétences » et "connaissances", commençons par mentionner un article de Behrent (2014) dans lequel il décrit le modèle conceptuel de l'école française et celui de l'école américaine. La première, d'inspiration durkheimienne, considère que "la connaissance doit cultiver une révérence pour les acquis culturels et scientifiques de l'humanité - la vérité, l'érudition, le patrimoine 
culturel» tandis que la seconde met en avant les individus "portés au savoir par les questions que suscite leur expérience quotidienne ». Il ne s'agit pas de vanter le modèle de telle ou telle nation (modèles par ailleurs critiqués par l'auteur), mais de relever que la distinction entre ces deux conceptions est marquée par la place de l'apprenant et de son objet. C'est en ce sens que Dubet (2014) rappelle le débat sur les différentes approches de la transmission dans l'éducation. Il soutient que «la formation intellectuelle doit conduire à l'émancipation, celle-ci étant comprise moins comme un épanouissement personnel que comme un accès à l'autonomie du jugement, condition de la citoyenneté " (p. 29). Ainsi, le rôle de l'enseignement consiste à répondre à des objectifs sur le très long terme. Selon Fedi (2014), trois modèles coexistent dans la pédagogie. Ils se distinguent par le rôle donné à l'enseignant et à l'apprenant : le modèle constructiviste qui naît à partir des années 1980 et qui place l'élève au centre de son apprentissage. Pour ce mouvement, "l'intelligence est une fonction d'adaptation liée aux expériences de l'individu et à son environnement social» (p. 29). Le modèle culturaliste quant à lui se concentre sur la «transmission d'une façon d'être et de penser sans laquelle il est difficile de se construire et s'orienter » (p. 30). Il se préoccupe de ce qui doit être enseigné et ce que l'apprenant doit connaître d'un monde qui lui "préexiste ». Enfin, le modèle utilitariste, qui a probablement généré le débat sur les compétences et les connaissances et qui, selon les auteurs, doit faire face à quelques problèmes qui appellent à privilégier l'aspect pluridisciplinaire des connaissances, notamment celui de la "décomposition» de compétences en éléments simples. Il semble, en effet, difficile de concevoir une tâche dans un domaine et un seul sans faire appel à un autre, mais aussi sans faire appel à des compétences antérieures (savoirs maîtrisés). Pour l'auteur, « la capacité d'adaptation des individus est souvent corrélée avec une solide formation intellectuelle, esthétique ou scientifique : la possession de savoirs désintéressés se révélerait finalement plus utile que la recherche de l'utilité immédiate ${ }^{2} »$ (p. 30). Selon Fabre (2015), la notion de compétence, qu'il considère comme un "fourre-tout ", se développe avec la linguistique chomskyenne (qui s'inscrit dans le paradigme logico-grammatical) où « elle désigne l'aptitude innée à comprendre et à émettre une infinité de phrases dans une langue donnée, les performances attestant de cette compétence sous-jacente ${ }^{3}$ ».

Mais le lien entre compétence et connaissance sous-tend selon Rastier (2013) un rapport entre théorie et pratique. L'auteur soutient que :

[...] comme les connaissances ont cédé la place aux compétences et que tout s'organise désormais en "niveaux de compétence », la dualité entre connaissances et compétence n'est pas problématisée, alors même qu'elle spécifie le rapport plus général entre la théorie et la pratique (p. 34).

La dénégation de cette dualité autorise, d'une part, le fixisme en matière de «représentation des connaissances" et d'autre part, elle obère toute dimension praxéologique de l'appropriation. Ce n'est pourtant que par sa pratique, livresque ou autre, et donc par son action singulière qu'un individu s'approprie des connaissances. Si bien que Hirtt (2009), dans la perspective comparative de l'approche constructiviste et de l'approche par compétence, met en relief la place et les finalités de l'apprentissage et la maîtrise des connaissances. Il affirme que :

Dans la pédagogie constructiviste, le plus important n'est pas que l'élève parvienne au bout de la tâche, mais qu'il ait mis à profit son travail (et ses erreurs éventuelles) pour progresser dans la découverte et la maîtrise des connaissances. Dans l'approche par compétences, le progrès dans la maîtrise des savoirs n'est pas un objectif en soi. Seul compte le résultat final (p. 23). 
La progression chez Hirtt (2009) n'est pas de répondre correctement aux objectifs d'une compétence mais de cheminer vers l'accomplissement de la tâche. C'est la réflexion et l'esprit critique qui sont mis en évidence dans l'approche constructiviste ${ }^{4}$.

11 C'est aussi ce que soutient Duru-Bellat (2012), pour qui les compétences ont conduit à «une machinerie évaluative qui a de quoi rendre perplexes les enseignants» sur le caractère abstrait des évaluations telles que «adapter sa communication en fonction du contexte ».

Tout semble être une question de méthode et d'approche par la réflexion, la sensibilisation et la responsabilisation. C'est dans la praxéologie ${ }^{5}$ entendue comme théorie de l'action, qui pose la question du rôle à attribuer à la pratique sous l'angle de l'agir humain, qu'il nous semble possible de renouveler les rapports entre apprenants et contenus. Ce point de vue amène à considérer le caractère actif et critique de toute appropriation de connaissance ${ }^{6}$.

\subsection{Notion de « compétences » en didactique des langues}

En didactique des langues, cette dualité est aussi perceptible lorsque sont évoquées la définition et la fonction de la langue. Guichon (2006, p. 18) soutient que la langue peut être considérée soit comme un système de signes (comme dans la tradition linguistique saussurienne) soit comme un ensemble de moyens qui servent à l'expression et la transmission des significations. Pour l'auteur, la langue est « un outil de transaction entre les individus », s'inspirant d'Ellis (2003) qui conçoit « une forme d'enseignement qui traite la langue d'abord comme un outil de communication plutôt que comme un objet d'étude ou de manipulation ${ }^{7} »$. Mais il nous semble que les connaissances de la langue passent par une étude de son lieu de production orale ou écrite, c'est-à-dire par le texte. Les informations socioculturelles qu'il comporte à la fois dans sa structure et dans ses conditions de production conduisent à voir le texte comme un objet d'apprentissage. Proposer le paradigme des connaissances amène donc à repenser le lien entre l'apprenant et son objet.

13 Les références citées plus haut montrent qu'il est indispensable de former à la responsabilité dans la durée plus qu'à l'efficacité à courte vue. Cet aspect est très présent dans les objectifs du CECR qui explique qu'il « décrit aussi complètement que possible ce que les apprenants d'une langue doivent apprendre afin de l'utiliser dans le but de communiquer; il énumère également les connaissances et les habiletés qu'ils doivent acquérir afin d'avoir un comportement langagier efficace ». Notre propos consiste à dire que la communication entre les personnes est, de fait, plus complexe dans la réalité, surtout entre cultures différentes. C'est pour cette raison que les connaissances s'adaptent mieux à un dispositif didactique où les objets sémiotiques ont toute leur place, notamment en tenant compte des pratiques discursives dans lesquelles s'inscrivent les textes.

\subsection{Le genre : l'outil/objet - repère des pratiques sociales en didactique}

Parce que le genre discursif s'inscrit dans une pratique sociale donnée dont il témoigne en retour, la reconnaissance et l'apprentissage des structures qui caractérisent le genre sont donc nécessaires en didactique des langues. Les contributions autour de la notion de 
genre et de types de discours en didactique ont foisonné depuis les années 1980. Il s'agira ici de considérer quelques-unes des études qui ont attiré notre attention dans le cadre de cet article. La plupart des chercheurs en didactique s'inspirent de la théorie bakhtinienne du genre (Beacco, 2004 ; Schneuwly \& Dolz-Mestre, 1997 ; Schneuwly, 2007) pour mettre à profit les divers concepts considérés. Pour Schneuwly et Dolz-Mestre (1997), les genres peuvent être des « outils qui fondent la possibilité de communication ». Ils s'appuient sur l'idée de «formes relativement stables» et évoquent des "rituels de pratiques langagières » et donc de pratiques sociales. Ces considérations sur le genre permettent par conséquent de l'envisager comme support didactique pour l'enseignement des pratiques langagières et donc des pratiques sociales. Les moyens qui relient les pratiques langagières et la didactique relèvent alors du genre comme « une composition d'outils relatifs à la structure, aux contenus et aux unités linguistiques» (Shneuwly, 2007). De même, Beacco (2007) définit le " genre de discours ou genre discursif ${ }^{8}$ » comme :

des formes prises par la communication telle qu'elle s'effectue dans une situation sociale et une communauté de communication données, identifiées comme telles par des paramètres (lieu, type de participants...) et où prend place une forme discursive spécifique comme: une conférence, un fait divers, une anecdote, une dispute, un mythe, une prière (pp. 96-97).

La connaissance des genres est donc non seulement importante pour la production ${ }^{9}$ mais également pour la compréhension en didactique des langues. Si les caractéristiques qui lui sont relatives sont familières au lecteur d'un texte, l'interprétation n'en sera que plus aisée. Cette notion a donc une influence sur le lecteur du texte, et agit comme un " horizon d'attente ${ }^{10}$ " qui réduit et définit les possibles interprétations qu'en fera le lecteur. C'est par le biais des normes qui singularisent les genres qu'il y aura anticipation et intercompréhension ${ }^{11}$.

\section{Expérimentation}

\subsection{Cadre théorique : sémantique interprétative assistée par ordinateur}

\subsubsection{Recherches en didactique sur corpus textuel}

Notre démarche rejoint plusieurs travaux se portant en faveur d'une didactique des langues sur corpus. Ces recherches ont intégré cette dimension où l'apprenant pouvait aborder les contenus textuels et y réfléchir. Johns (1990), qui a impulsé la démarche en didactique, voyait en tout apprenant un "Sherlock Holmes ». Dans une recherche plus récente, les travaux de Chachkine et al. (2013) évoquent la notion d'« apprenant réflexif ». Ces études utilisent souvent des outils d'analyse des textes pour permettre aux apprenants de décrire et d'apprendre la langue via une méthode déductive et par euxmêmes (en comparant des contextes, par exemple). Des chercheurs tels que Chambers $(2005,2007)$, Kerr (2013) et Landure (2013) ont développé l'approche en français langue étrangère, Johns (1991) en anglais et Schaeffer-Lacroix (2014) et Vyatkina (2016) en allemand. Mais aucune recherche ne s'est jusqu'ici intéressée à l'intégration de l'étude interprétative des textes avec des outils de données statistiques textuelles. 


\subsubsection{Sémantique de corpus}

17 C'est donc en nous appuyant sur ces travaux que nous faisons le choix de la sémantique de corpus, c'est-à-dire de l'utilisation conjointe des outils de la sémantique interprétative (Rastier, 2001a) et des outils de statistiques textuelles. Ceux-ci permettent en effet de mettre en évidence des régularités et des différences entre corpus, que la lecture linéaire pourrait difficilement constater. Les calculs statistiques intégrés au logiciel Lexico 3 (Salem et al., 2003) qui supporte le traitement de plusieurs langues renferment plusieurs fonctionnalités comme les concordances ${ }^{12}$ et les spécificités. Ces dernières peuvent en effet aider par exploration ciblée à l'identification des éléments langagiers les plus caractéristiques d'un texte à l'autre.

Pour comprendre l'analyse interprétative transposée en contexte didactique, prenons l'énoncé suivant issu de notre corpus journalistique et intéressons-nous au lexème «alJazeera » (chaîne d'information qatarie) :

$$
\begin{aligned}
& \text { الجزيرة لشبكةأوباماباراك الأميركي الرئيس إدارةقبل من توددمحاولات الأخيرةالأسابيعشهدت « }
\end{aligned}
$$

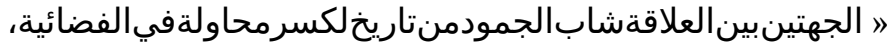

«L'administration du président Barack Obama et la chaine al-Jazeera ont tenté ces dernières semaines un rapprochement dans l'objectif de sortir de l'impasse historique qui caractérise la relation entre les deux partis. »

19 La signification d'un mot peut être remodelée par des opérations interprétatives: l'activation, l'inhibition ou la propagation.

- L'activation actualise les sèmes afférents. Dans l'exemple ci-dessus, le contexte active le sème /institution politique/ dans le lexème " al-Jazeera ».

- L'inhibition empêche l'actualisation de sèmes inhérents qui sont alors dits "virtualisés ». Toujours dans le même extrait, les sèmes /média/, /objectivité/ sont inhibés par le contexte, puisque dans l'énoncé, al-Jazeera prend des dispositions avec l'administration américaine pour normaliser leurs relations.

- Enfin, la propagation qui concerne les sèmes afférents contextuels est l'opération qui permet ici de propager le sème /État/ sur « al-Jazeera ». Le sème est afférent contextuel car «al-Jazeera » a pris la figure d'un média partial et interventionniste auprès des lecteurs et téléspectateurs.

\subsection{Corpus de l'expérimentation}

Pour le public d'apprenants arabisants, nous avons choisi un thème largement relayé dans les médias arabes: les révolutions en Égypte. Pour ces étudiants, ce foisonnement est captivant mais aussi déstabilisant puisque le flux informationnel est rapide et peut paralyser l'attention et le jugement du lecteur face à la multitude de termes associés aux événements. Face à cette abondance qui caractérise le visage des médias aujourd'hui, l'expérimentation se propose de tenir compte de ce type de contenu et de problématique en classe.

21 Le corpus utilisé est un ensemble de textes provenant de trois journaux différents en ligne (deux panarabes - saoudien et qatari - et un national égyptien. Il a été sélectionné par mots-clés et traite de la révolution en Égypte en 2011. Pour sa constitution, nous avions adopté la définition de la sémantique des textes, à savoir «un regroupement structuré de textes intégraux, documentés, éventuellement enrichis par des étiquetages, 
et rassemblés : (i) de manière théorique réflexive en tenant compte des discours et des genres, et (ii) de manière pratique en vue d'une gamme d'applications » (Rastier, 2004). La sélection des articles a été effectuée à partir de trois mots-clés directement entrés sur les moteurs de recherche des journaux : "protestation », «manifestation » ainsi que le nom du pays concerné : « Égypte ». Le choix de ne pas sélectionner le mot « révolution » était délibéré. En effet, les articles relataient tous des manifestations ou des protestations, mais sans pour autant employer le terme « révolution » dans tous les journaux.

Tableau 1. Données du corpus

\begin{tabular}{|l|l|l|l|}
\hline Journaux & Al-Jazeera & Al-Sharq al-Awsat & Al-Masry al-Youm \\
\hline Nombre d'articles & 37 articles & 44 articles & 71 articles \\
\hline
\end{tabular}

\subsection{Déroulement des séquences}

L'expérimentation s'est déroulée sur une période de sept semaines (une heure trente par séance). Pour des raisons relatives aux disponibilités des participants, nous avons constitué deux groupes : le premier de deux participants étudiants en licence 2, le second de cinq participants de niveaux différents (de licence 3 à master 2). Nous avons travaillé essentiellement sur le rapport texte-lecteur. Nous avons eu le rôle de médiateur à l'oral et en interaction avec les apprenants pour clarifier des questions ou rendre compréhensible ce qui était demandé. Il y avait beaucoup de discussions pendant les séances, voire des débats et il fallut recentrer le sujet pour ne pas perdre de vue l'objectif de la séquence. La progression des séquences a été un facteur important pris en compte pour ne pas dérouter les participants et établir une cohérence dans le développement et la progressivité de l'expérimentation.

23 Le tableau 2 ci-dessous indique l'articulation entre les input résultant des statistiques du logiciel d'analyse de corpus Lexico 3 et les notions de la sémantique interprétative. Autrement dit, il éclaire la combinaison entre les outils qualitatifs et les outils quantitatifs, et leur complémentarité dans une perspective didactique.

Les séquences 1, 2 et 3 ont pour input un seul et même texte. Les différentes concordances ont été mises à disposition de l'apprenant au fur et à mesure des séquences. À partir de la séquence 4 , les étudiants ont accès à l'ensemble du corpus « Révolution Égypte ».

Tableau 2. Description des séquences

\begin{tabular}{|l|l|l|l|}
\hline Séquence & Input & Notions sémantiques & Output demandé \\
\hline 1 & Texte et concordances & $\begin{array}{l}\text { Sèmes inhérents et } \\
\text { afférents contextuels }\end{array}$ & $\begin{array}{l}\text { Réponses sur le sens des } \\
\text { mots en contexte }\end{array}$ \\
\hline 2 & Texte et concordances inhérents et & $\begin{array}{l}\text { Réponses sur le sens des } \\
\text { mots en contexte ; } \\
\text { sémantiques }\end{array}$ & $\begin{array}{l}\text { Traductions ; } \\
\text { Reformulations }\end{array}$ \\
\hline
\end{tabular}




\begin{tabular}{|c|c|c|c|}
\hline 3 & Texte et concordances & $\begin{array}{l}\text { Sèmes inhérents et } \\
\text { afférents, classes } \\
\text { sémantiques }\end{array}$ & $\begin{array}{l}\text { Écriture d'un texte } \\
\text { d'opinion dans le } \\
\text { « courrier des lecteurs " }\end{array}$ \\
\hline 4 & $\begin{array}{l}\text { Deux vidéos et corpus } \\
\text { «Révolution Égypte» }\end{array}$ & $\begin{array}{l}\text { Sèmes afférents } \\
\text { contextuels }\end{array}$ & $\begin{array}{l}\text { Réponses à des questions } \\
\text { d'analyse; Traduction de } \\
\text { phrases en français }\end{array}$ \\
\hline 5 & $\begin{array}{l}\text { Contextes larges d'une } \\
\text { occurrence apparaissant } \\
\text { dans les articles d'opinion } \\
\text { et d'information }\end{array}$ & $\begin{array}{lr}\text { Sèmes } & \text { afférents } \\
\text { contextuels } & \text { et } \\
\text { socionormés ; } & \text { univers } \\
\text { énonciatif (dialogique) }\end{array}$ & $\begin{array}{l}\text { Réponses à des questions } \\
\text { d'analyse en français et } \\
\text { en arabe }\end{array}$ \\
\hline 6 & $\begin{array}{l}\text { Corpus } \begin{array}{l}\text { "Révolution } \\
\text { Égypte " et tableaux de } \\
\text { spécificités }\end{array}\end{array}$ & $\begin{array}{l}\text { Classes sémantiques; } \\
\text { Intertextualité ; } \\
\text { Architextualité }\end{array}$ & $\begin{array}{l}\text { Réponses à des questions } \\
\text { d'analyse ; Formulation } \\
\text { d'hypothèses }\end{array}$ \\
\hline 7 & \begin{tabular}{lll} 
Corpus & \multicolumn{2}{c}{ "Révolution } \\
Égypte ", tableaux de \\
spécificités et article en \\
français
\end{tabular} & $\begin{array}{l}\text { Classes sémantiques ; } \\
\text { Intertextualité ; } \\
\text { Architextualité }\end{array}$ & $\begin{array}{l}\text { Réponses à des questions } \\
\text { en français }\end{array}$ \\
\hline
\end{tabular}

\subsection{Production des étudiants}

\section{Extrait des réponses de la séquence 1 : Expliciter les différences d'acception d'un mot dans un même texte}

Dans la séquence 1, nous souhaitions montrer l'incidence sémantique des co-occurrents. L'une des tâches était d'observer les différents «mots voisins » du mot ثورة (révolution) puis de comprendre s'il avait le même sème dans tout le texte. Voici les réponses des trois étudiants en L2 à leurs observations suite à la question : «Selon vous, est-ce que "ثورة" a le même sens tout le long du texte ? Pourquoi ?»

Séverine (L2) :

On voit que selon ses co-occurrents, le sens du mot change. Il désigne dans les premières occurrences l'idée d'une révolution populaire en vue d'un changement social ou politique, alors qu'en fin de texte le mot désigne plutôt un changement brutal dans le domaine technologique. Ceci permet de créer un parallèle, voire de donner l'idée d'une complémentarité, entre les soulèvements et l'émergence des nouvelles technologies de communication, en appuyant ainsi sur le rôle crucial que ces dernières ont joué.

À ce stade, Séverine observe le contexte global du mot (le texte en entier) et les concordances du mot « révolution ». Elle peut observer les unités de sens superposées à chaque occurrence dans la fenêtre de concordance. Elle a ainsi pu faire une observation rapide et ciblée du contexte, analyser les associations et formuler des hypothèses sur le sens du mot.

Nassim (L2) :

Non, le sens dépend principalement du contexte et des co-occurrences qui l'entourent. Le titre du texte, où le terme est cité, évoque à la fois l'idée de «Révolutions arabes » faites par Internet mais aussi du progrès des moyens de 
communication qui ont permis cela. Deux fois le terme désigne clairement la révolution en tant que mouvement populaire. Les autres fois il s'agit plutôt de dérision ou pour insister sur le fait que c'est une "révolution du progrès technique » qui a permis ces révolutions. Il va jusqu'à qualifier les conflits de générations de "révolution des générations». Le sens général du terme reste cependant plus ou moins le même, c'est l'idée d'un changement ou bouleversement, ici vécu par l'auteur comme négatif.

L'apprenant saisit ici les différentes nuances du terme en fonction de son contexte d'apparition; ce qui lui permet de faire une déduction plus étendue du sens et de comprendre la teneur du propos du journaliste («changement ou bouleversement, ici vécu par l'auteur comme négatif »).

Katia (L2) :

Non, tout au long du texte, ce mot prend des significations différentes qui peuvent être au sens propre ou au sens figuré. Dans l'article, lorsqu'il est suivi d'un nom de pays, il est souvent au sens propre, c'est-à-dire qu'il signifie révolution dans le sens d'un groupe de personnes ou peuple qui se révolte contre une force qui l'oppresse.

Tous ont remarqué les afférences contextuelles, en reprenant le vocabulaire linguistique (« significations", " co-occurrents»), parfois un peu inexact («sens propre», "sens figuré »). Leurs analyses sont intéressantes : Séverine parle de "créer un parallèle, voire de donner l'idée de complémentarité » entre les deux significations portées par " révolution ». Les étudiants ont pu, par une simple observation ciblée, comprendre rapidement qu'un même mot peut avoir plusieurs sens.

\section{Extrait des réponses de la séquence 5 : Explicitation du lien entre syntaxe, sémantique et dialogique en comparant plusieurs textes}

Dans la séquence 5, il a été demandé de comparer l'emploi des temps entre des extraits issus d'articles d'information et d'opinion. L'objectif était de montrer la façon dont les régularités morphosyntaxiques peuvent être plus facilement observées par une analyse contrastive des genres. Il s'agit ici de tester l'une des caractéristiques du genre textuel : «Aucun texte n'est écrit uniquement dans une langue ; il est écrit dans un genre dans un discours, prenant évidemment en compte les contraintes d'une langue » (Rastier, 2005, p. 33) et d'observer ces «contraintes" de langage par le principe d'intertextualité (comme relation entre deux textes, autrement dit une relation d'interdépendance sémantique entre deux textes).

Les participants ont reçu deux sous-corpus de deux genres différents (articles d'opinion et articles d'information). Pour cette séquence, nous avons demandé aux étudiants d'observer le contexte du mot «Internet » :

31 Aymen (L3) :

Dans les extraits information, les verbes sont à l'accompli et précèdent souvent des déclarations ou des citations; alors que dans les extraits opinion, nous avons plus de variantes entre l'accompli et l'inaccompli car le deuxième mode permet à l'auteur de faire des constations, de signaler des habitudes, et d'affirmer des vérités générales.

Séverine (L2) :

Les verbes de la première rubrique sont plutôt neutres et informatifs, de vérité générale; alors que l'on ressent en ceux de la deuxième rubrique une forte affirmation, ils sont aussi plus descriptifs. On peut en conclure que la forme des 
énoncés de la première rubrique est objective alors que la forme des énoncés de la

deuxième rubrique est plus argumentative.

33 Ainsi, les deux étudiants ont remarqué des différences d'emploi des verbes et des temps. Séverine ne précise pas les temps qu'elle rencontre dans les textes mais voit une différence qu'elle tente de décrire. La progression de la séquence leur a permis de pointer une corrélation entre temps employé et genre discursif. Cette réflexion commence à émerger à partir du moment où il leur est demandé de comparer les extraits entre eux. En effet, les caractéristiques morphosyntaxiques sont mises en évidence par le phénomène d'intertextualité que permet le logiciel.

34 Les positions énonciatives sont ici marquées par l'usage du temps verbal (ou aspect), lié à l'intention de l'énonciateur. Dans les articles d'information, les verbes sont à l'accompli et décrivent un événement déjà passé (ils sont souvent accompagnés d'adverbes de temps passé) tandis que les articles d'opinion sont inscrits dans la réflexion, et font un usage varié des temps verbaux. Ces constatations sont fondées sur la fréquence des temps à l'accompli ou à l'inaccompli dont l'usage est repéré par les étudiants.

\subsection{Discussions}

Cette étude dont nous n'avons pu expliciter que deux séquences et leurs résultats montre le potentiel des outils d'analyse de corpus et de sensibilisation à la métalinguistique pour la compréhension et l'expression écrite. Certains étudiants ont déclaré dans le questionnaire distribué en fin d'expérimentation que leurs capacités de lecture et de compréhension en langue arabe se sont améliorées. Des problèmes ont aussi été soulevés, notamment sur la méthode didactique: il semble en effet que pour les étudiants de licence, il soit plus aisé de travailler d'abord en lecture ciblée avant d'aborder le texte dans sa globalité. Ils ont tout de même réussi à comprendre les nuances sémantiques des expressions étudiées. Les étudiants en master, eux, sont à l'inverse plutôt frustrés lorsque les input ne sont que des concordances avec peu de mots qui entourent le terme cible dont il faut étudier la teneur sémantique. Pour tous, c'était la première fois qu'ils travaillaient sur la comparaison simultanée des textes que permet le logiciel.

Cette première expérimentation en pratique d'analyse critique sur un corpus arabe est donc positive, puisqu'elle permet aussi d'affiner la méthode en fonction du public d'apprenants et de proposer d'autres séquences sur d'autres thématiques.

\section{Conclusion}

Cette expérience textuelle menée sur le terrain de la didactique des langues nous semble illustrer notre propos sur l'enjeu d'une praxis textuelle. Cette dernière est une condition nécessaire à l'interprétation des textes si l'on appréhende, comme le préconise la sémantique interprétative, le texte comme une suite linguistique attestée au sein d'une pratique. Elle illustre le fait que l'on ne traduit jamais de langue à langue mais des textes comme autant de pratiques culturelles. En rompant avec une vision atomiste et utilitariste des langues, l'approche textuelle, comprise comme performance complexe constitutive du milieu sémiotique dans lequel nous vivons, est, selon nous, la voie de résistance à l'ethos néolibéral et ses portefeuilles de compétences qui s'impose aujourd'hui dans le milieu de l'éducation. L'accès aux corpus numériques, couplé aux logiciels de statistiques textuelles (tel Lexico 3), autorise de nouvelles méthodes 
d'interprétation (domaine de l'herméneutique numérique). Il est d'autant plus nécessaire que les chercheurs puissent répondre concrètement aux exigences de notre temps en matière de flux informationnel souvent contradictoire. Les outils théoriques et pratiques peuvent facilement s'intégrer dans les curriculum de langue ou de sciences de la communication. C'est en ce sens que Wolton (2009, p. 72) soutient que « l'information est toujours la condition de l'esprit critique » et que, « quelle que soit par ailleurs la nature de l'information, le rôle du récepteur s'impose ». L'enjeu est de fournir les clés pour la responsabilisation et la conscientisation des données langagières.

\section{BIBLIOGRAPHIE}

Beacco, J.-C. (2004). Trois perspectives linguistiques sur la notion de genre discursif. Langages, 1, 109-119.

Beacco, J.-C. (2007). L'approche par compétences dans l'enseignement des langues. Paris : Didier.

Behrent, M. (2014). Instruire ou éveiller ? Un débat transatlantique. Sciences humaines, 263, 20-20.

Cassirer, E. (1991). Logique des sciences de la culture (trad. de Zur Logik der Kulturwissenschaften, Göteborg : Göteborgs Högskolas Årsskrift 47, 1942). Paris : Éditions du Cerf.

Dubet, F. (2014). Heur et malheur de l'école républicaine. Sciences humaines, 263, 28-31.

Duru-Bellat, M. (2012). Connaissances ou compétences, que transmettre ? Les grands dossiers des Sciences humaines, 27(6), 12-12. Repéré à https://www.cairn.info/magazine-les-grands-dossiersdes-sciences-humaines-2012-6-page-12.htm

Ellis, R. (2003). Task-based language learning and teaching. Oxford : University Press Oxford.

Fabre, M. (2015). Généalogie de l'éthos néo-libéral. Perspectives foucaldiennes. Dans M. Fabre et C. Gohier (dir.), Les valeurs éducatives au risque du néo-libéralisme (pp. 91-103). Rouen : PURH.

Fedi, L. (2014). Quel rôle pour l'enseignant? Sciences humaines, 263, 30.

Guichon, N. (2006). Langues et TICE : méthodologie de conception multimédia. Paris : Éditions Ophrys.

Hirtt, N. (2009). L'approche par compétences : une mystification pédagogique. L'école démocratique, 39, 1-34. Repéré à http://www.skolo.org/2009/10/01/lapproche-par-competences-unemystification-pedagogique/

Johns, T. (1990). From printout to handout: Grammar and vocabulary teaching in the context of data-driven learning. CALL, 10, 14-34.

Johns, T. (1991). Should you be persuaded: Two samples of data-driven learning materials. English Language Research Journal, 4, 1-16.

Kerr, B. (2013). Grammatical description and classroom application: Theory and practice in datadriven learning. Bulletin suisse de linguistique appliquée, 97, 17-39.

Landure, C. (2013). Corpus et dictionnaires : prise de conscience linguistique chez des apprentis LANSAD. Bulletin suisse de linguistique appliquée, 97, 59-76. 
Makouar, N. (2014). Sémantique de corpus et didactique des langues : application à des discours journalistiques et politiques de langue arabe (Thèse de doctorat). Paris : Inalco.

Mauss, M. (1924). Essai sur le don. Forme et raison de l'échange dans les sociétés archaïques. L'Année sociologique (1896/1897-1924/1925), 1, 30-186. DOI : http://dx.doi.org/doi:10.1522/ cla.mam.ess3

Privat, J.-M. \& Vinson, M.-C., (1988). Tableaux de genres : travailler les critères de genre en lecture-écriture. Pratiques, 59, 3-17.

Rastier, F. (2001). Arts et sciences du texte. Paris : PUF.

Rastier, F. (2003). Le langage comme milieu : des pratiques aux œuvres. Texto! (Rubrique Dits et inédits.) Repéré à http://www.revue-texto.net/1996-2007/Inedits/Rastier/Rastier_Langage.html (consulté le 15 mars 2017).

Rastier, F. (2006). De la signification lexicale au sens textuel : éléments pour une approche unifiée. Texto ! Repéré à http://www.revue-texto.net/Inedits/Rastier/Rastier_Significationlexicale.html (consulté le 15 mars 2017).

Rastier, F. (2013). Apprendre pour transmettre : l'éducation contre l'idéologie managériale. Paris : PUF.

Rastier, F. (2015). Saussure au futur. Paris : Les Belles Lettres

Rastier, F. (2017). De la sémantique structurale à la sémiotique des cultures. Actes sémiotiques, 120. Repéré à http://epublications.unilim.fr/revues/as/5734

Rickert, H. (1899). Kulturwissenschaft und Naturwissenschaft (trad. de l'allemand par C. Prompsy et M. de Launay). Freiburg : J.C.B. Mohr.

Salem, A. et al. (2003). Lexico3 : Outils de statistique textuelle. Manuel d'utilisation. Lexi\&co. DOI : http://lexi-co.com/ressources/manuel-3.41.pdf

Saussure, F. D. (2002). Écrits de linguistique générale (texte établi et édité par S. Bouquet et R. Engler). Paris : Gallimard.

Schaeffer-Lacroix, E. (2014). Impact de discussions métalinguistiques sur l'apprentissage de la production écrite en allemand, langue étrangère. Linx-Revue des linguistes de l'université Paris Ouest Nanterre La Défense, 72, 193-211.

Schneuwly, B. \& Dolz-Mestre, J. (1997). Les genres scolaires des pratiques langagières aux objets d'enseignement. Repères : recherches en didactique du français langue maternelle, 15, 27-40 (M. Dabène et G. Ducancel (dir.), « Pratiques langagières et enseignement du français à l'école »). Repéré à https://archive-ouverte.unige.ch/unige:32530

Schneuwly, B. (2007). Genres écrits et oraux et forme scolaire. Dans C. Boré (dir.), Construire et exploiter des corpus de genres scolaires (Diptyque, 10, pp. 13-26). Namur : Presses universitaires de Namur.

Vyatkina, N. (2016). Data-driven learning for beginners: the case of German verb-preposition collocations. ReCALL, 28(02), 207-226.

Wolton, D. (2009). Informer n'est pas communiquer. Paris : CNRS éditions.

\section{NOTES}

1. Glossaire de la sémantique interprétative disponible sur http://www.revue-texto.net/ 
2. Ce qui rappelle l'analyse de Todorov (cité par Privat \& Vinson, 1988) pour qui « le travail de connaissance vise une vérité approximative, non une vérité absolue. [...] L'imperfection est, paradoxalement, une garantie de survie » (Introduction à la littérature fantastique).

3. Fabre, 2015, p. 97

4. Selon les chercheurs en éducation, la démarche doit redonner à la connaissance ses droits et son accès par une pédagogie où les apprenants participent à la construire. Pour Duru-Bellat (2012) : «La philosophe Martha Nussbaum dénonce une éducation "tournée vers le profit". Elle promeut au contraire une éducation à la démocratie, développant l'indépendance d'esprit, l'imagination et la capacité d'empathie, et fondée pour ce faire sur les humanités et les arts. Il ne s'agit pas de promouvoir une discipline de plus, mais un état d'esprit, en invitant les élèves à se mettre dans la peau d'un personnage de roman, à analyser leurs émotions devant une œuvre d'art, à s'étonner de si bien comprendre les propos d'un auteur - philosophe ou politique datant de plusieurs siècles... »

5. La praxéologie entendue comme théorie de l'action par le sociologue Alfred Espinas (1890), proche de Durkheim, qui souhaitait enrichir la téchnē ( $\tau \dot{x} \chi \vee \eta)$, ou compétence pratique à l'image du geste de l'artisan, au logos, compris comme connaissances acquises parce que transmises à partir de ces mêmes compétences. Espinas, A. (1890). Les origines de la technologie, Revue philosophique, XV année, XXX, pp. 114-115.

6. La connaissance est action singulière, elle ne se fige en aucun de nous puisqu'elle n'est connaissance que reçue par nous: ici l'apprenant qui l'introduit dans son vécu perceptif (couplage avec l'environnement culturel que nous façonnons autant qu'il nous façonne).

7. Cité par Guichon (2006, p. 18).

8. Il emploie le terme "genre de discours " préférentiellement à celui de "type de texte " (narratif, descriptif, injonctif...) qui n'a pas su élaborer, selon lui, une description des classes de textes (Beacco, 2007, p. 97).

9. «Sur le versant de l'usage et de l'apprentissage, le genre peut ainsi être considéré comme un méga-outil qui fournit un support à l'activité dans les situations de communication et un repère pour les apprenants » (Schneuwly \& Dolz-Mestre, 1997, p. 29).

10. «Le texte nouveau évoque pour le lecteur (ou l'auditeur) tout un ensemble d'attentes et de règles du jeu avec lesquelles les textes antérieurs l'ont familiarisé et qui, au fil de la lecture, peuvent être modulées, corrigées, modifiées ou simplement reproduites » (Jauss, 1978, p. 51).

11. Rastier rappelle également que «l'analogie des pratiques et celle des genres qui en découle permet la traduction voire tout simplement l'intercompréhension » (Rastier, 2003, p. 36).

12. Méthode d'extraction textuelle basée sur la présentation d'extraits textuels contenant tous le même mot ou la même séquence, le même motif.

\section{RÉSUMÉS}

Dans cet article nous discutons l'apport de la sémantique des textes en éducation et en didactique des langues. Il est question de saisir les enjeux d'une praxéologie textuelle impliquant une rupture avec la vision utilitariste de la langue. Après avoir rappelé notre ancrage théorique, nous proposons de revenir sur la notion de «compétences » qui est au centre des préconisations du Cadre européen commun de référence pour les Langues, compromettant la démarche critique de l'individu via une catégorisation fermée des aptitudes langagières à acquérir. Nous illustrerons 
notre propos théorique à travers une expérimentation pour intégrer une démarche critique des textes en classe de langue.

In this article we discuss the contribution of the Textual Semantics theory in education and in Second Language Acquisition. The aim is to apprehend textual praxeology challenges that involve a break with the utilitarian vision of language. After describing our theoretical framework, we propose to study the notion of "competences", which is at the heart of the Common European Framework of Reference for Languages' recommendations; a notion that compromises the critical approach of the individual through a closed categorization of language skills to be acquired. We will illustrate our theoretical point of view through an experimentation for the integration a critical approach of texts in language learning class.

\section{INDEX}

Mots-clés : sciences de la culture, textométrie, genre textuel, praxéologie, compétences/ connaissances

Keywords : cultural sciences, textometry, textual genre, praxeology, competence/knowledge

\section{AUTEURS}

\section{NADIA MAKOUAR}

Chercheuse associée-ERTIM, INALCO

\section{MARYVONNE HOLZEM}

Maître de conférences-Dylis \& LITIS, Université de Rouen Normandie 